\title{
Skin care management in cancer patients: an evaluation of quality of life and tolerability
}

\author{
Ann Cameron Haley • Cara Calahan • Mona Gandhi • \\ Dennis P. West • Alfred Rademaker • \\ Mario E. Lacouture
}

Received: 21 January 2010 / Accepted: 23 February 2010 /Published online: 25 March 2010

(C) The Author(s) 2010. This article is published with open access at Springerlink.com

\begin{abstract}
Purpose The objective of this study is to evaluate quality of life (QoL) and tolerability of three articles specifically developed for cancer skin care management (skin moisturizer, face moisturizer, and face wash).

Methods Participants were cancer patients $(n=99)$ receiving systemic anticancer therapies and/or radiotherapy at Northwestern University. Subjects were assessed at the initial visit for adverse skin reactions based on the National Cancer Institute's Common Terminology Criteria for Adverse Events version 3.0 and completed the Skindex-16 questionnaire, a self-reported dermatology-specific QoL instrument. All subjects were provided with three test articles and were instructed to use each test article once daily for 4 weeks. At the 4 -week follow-up $(n=77)$, the Skindex-16 was readministered, adverse skin reactions were assessed, and tolerability questionnaires were administered for each article used.
\end{abstract}

A. C. Haley $\cdot$ M. Gandhi $\cdot$ D. P. West $\cdot$ M. E. Lacouture $(\bowtie)$

Department of Dermatology,

Northwestern University Feinberg School of Medicine,

Chicago, IL 60611, USA

e-mail: lacoutum@mskcc.org

C. Calahan • M. E. Lacouture

SERIES Clinic, Northwestern Memorial Faculty Foundation, Northwestern University Feinberg School of Medicine,

Chicago, IL 60611, USA

D. P. West $\cdot$ M. E. Lacouture

Robert H. Lurie Comprehensive Cancer Center, Northwestern University Feinberg School of Medicine,

Chicago, IL 60611, USA

\section{A. Rademaker}

Department of Preventive Medicine,

Northwestern University Feinberg School of Medicine,

Chicago, IL 60611, USA
Results Dry skin, hand-foot skin reaction (HFSR), and skin rash (dermatitis) decreased significantly from baseline to follow-up. Presence of nail changes, skin rash (desquamation), and acne/acneiform eruptions did not significantly change from baseline. Subjects had a significantly lower mean overall Skindex-16 score at 4-week follow-up when compared to baseline. Most patients rated their overall experience with each test article as good or very good (highest rating).

Conclusion Skin care in cancer patients is suboptimal in part due to a lack of products and knowledge specific for this population. Our findings suggest that QoL improves with test article use, all of which were rated as good/very good for tolerability. Moreover, skin toxicity as manifested by dry skin, hand-foot skin reaction, and skin rash (dermatitis) were decreased with use of test articles within 4 weeks.

Keywords Skin care - Chemotherapy · Cancer - Dry skin . Radiation therapy $\cdot$ Skin irrations $\cdot$ HFSR

\section{Introduction}

Patients with various solid and hematologic tumors are surviving longer and are reporting an enhancement in quality of life $(\mathrm{QoL})$ with improved anticancer treatments [1]. However, dermatologic toxicities are noteworthy due to their high frequency and association with negative symptomatology [2,3]. Moreover, clinicians and patients report that dermatologic toxicities have a negative effect on patients' physical, functional, emotional, and social wellbeing [4]. Whereas skin irritation, hair changes, facial flushing/erythema, and dry skin are common skin reactions that negatively affect these patients; what may often be perceived as relatively minor complaints of dry skin, nail 
changes, and itching are also shown to negatively affect QoL, at least partly due to their unanticipated nature [5]. Failure to identify and treat these untoward events often leads to noncompliance or inconsistent administration of anticancer treatments, all of which may affect clinical outcome [3, 6, 7]. Consequently, it may often be crucial for patients undergoing anticancer therapy to proactively initiate skin care management in order to minimize the impact of commonly occurring toxicities $[8,9]$.

Current approaches to skin care management vary widely and are often based on anecdotal data or poorly controlled studies. Consequently, one approach has been to simply recommend that patients moisturize twice daily with thick emollients [9]. Gentle skin cleansing and maintenance of hydration of compromised skin may also be an important element of skin care management during cancer therapies. Some guidelines, for example, indicate washing with mild soap and water as routine care for patients receiving radiation therapy [10]. In a review of two trials assessing skin washing, both trials found less severe skin reactions to radiation therapy in washing groups $[9,11]$. Various vehicles such as lotions, creams, and ointments may be recommended, but clear evidence supporting one product form over another is lacking [10]. Products that are specifically created for skin care management during cancer treatment in patients who may suffer from dermatologic side effects are continuously being sought to address this unmet need [12].

Since there is insufficient data to evaluate the effectiveness and tolerability of interventions for cutaneous reactions resulting from anticancer therapies [10], the primary objective of this study is to evaluate QoL as well as tolerability of three products developed specifically for skin care management (skin moisturizer, face moisturizer, and face wash) during cancer treatments. The secondary objective of this study is to determine whether a correlation exists between patient tolerability with skin care management and severity of skin toxicity based on the National Cancer Institute's Common Terminology Criteria for Adverse Events (CTCAE) version 3.0. The CTCAE is a standard tool used by clinicians to report cancer treatment toxicities [13]. We hypothesized that cancer patients with CTCAE grades 0 or 1 skin toxicity will report improved QoL if products specifically designed for skin care management during cancer treatment are utilized.

\section{Methods}

Participants in this study were diagnosed cancer patients receiving cytotoxic chemotherapy, targeted or hormonal treatments, and/or radiotherapy at the Robert H. Lurie Comprehensive Cancer Center at Northwestern University between April and July 2009. A total of 99 subjects participated (Table 1). The study was approved by the Northwestern University Institutional Review Board, and all participants provided written informed consent prior to participation.

Subjects who agreed to participate were assessed at the initial visit for adverse skin reactions and graded based on the NCI-CTCAEv3.0 scale and, in addition, completed the Skindex-16 questionnaire, a self-reported dermatologyspecific QoL instrument that has undergone reliability and validity testing in other dermatology conditions such as acne and psoriasis [14]. All subjects were provided with three test articles specifically formulated for cancer skin care management: test article A (skin moisturizer), test article B (face moisturizer), and test article C (face wash). Test articles have been developed based on surveys conducted in cancer patients for preferences of skin products (Lindi ${ }^{\circledR}$ Skin, Philadelphia, PA). Subjects were instructed to use each test article once daily for 4 weeks and to record in a diary the date and time of each test article application in order to assess level of compliance. Subjects were interviewed by telephone to assess level of compliance at week2. At the 4-week follow-up interview, participants completed a follow-up Skindex-16 questionnaire, study personnel assessed cancer treatment-related skin toxicity according to NCI-CTCAEv3.0 criteria, and a cancer skin care management tolerability questionnaire (Fig. 1) was administered for each test article used.
Table 1 Demographic and clinical characteristics

HFSR hand-foot skin reaction

\begin{tabular}{llll}
\hline Characteristic & $\%$ Baseline $(n=99)$ & $\%$ 4-week follow-up visit $(\mathrm{n}=74-75)$ & $p$ value \\
\hline Sex & 1 & & \\
$\%$ males & 1 & \\
Presence of skin toxicity & & 21 & $<0.0001$ \\
Dry skin & 69 & 5 & 0.99 \\
Nail changes & 5 & 0 & 0.15 \\
Rash/desquamation & 3 & 7 & 0.32 \\
Acne/acneiform & 4 & 2 & 0.025 \\
Rash: dermatitis & 8 & 4 & 0.021 \\
HFSR & 15 & & \\
\hline
\end{tabular}




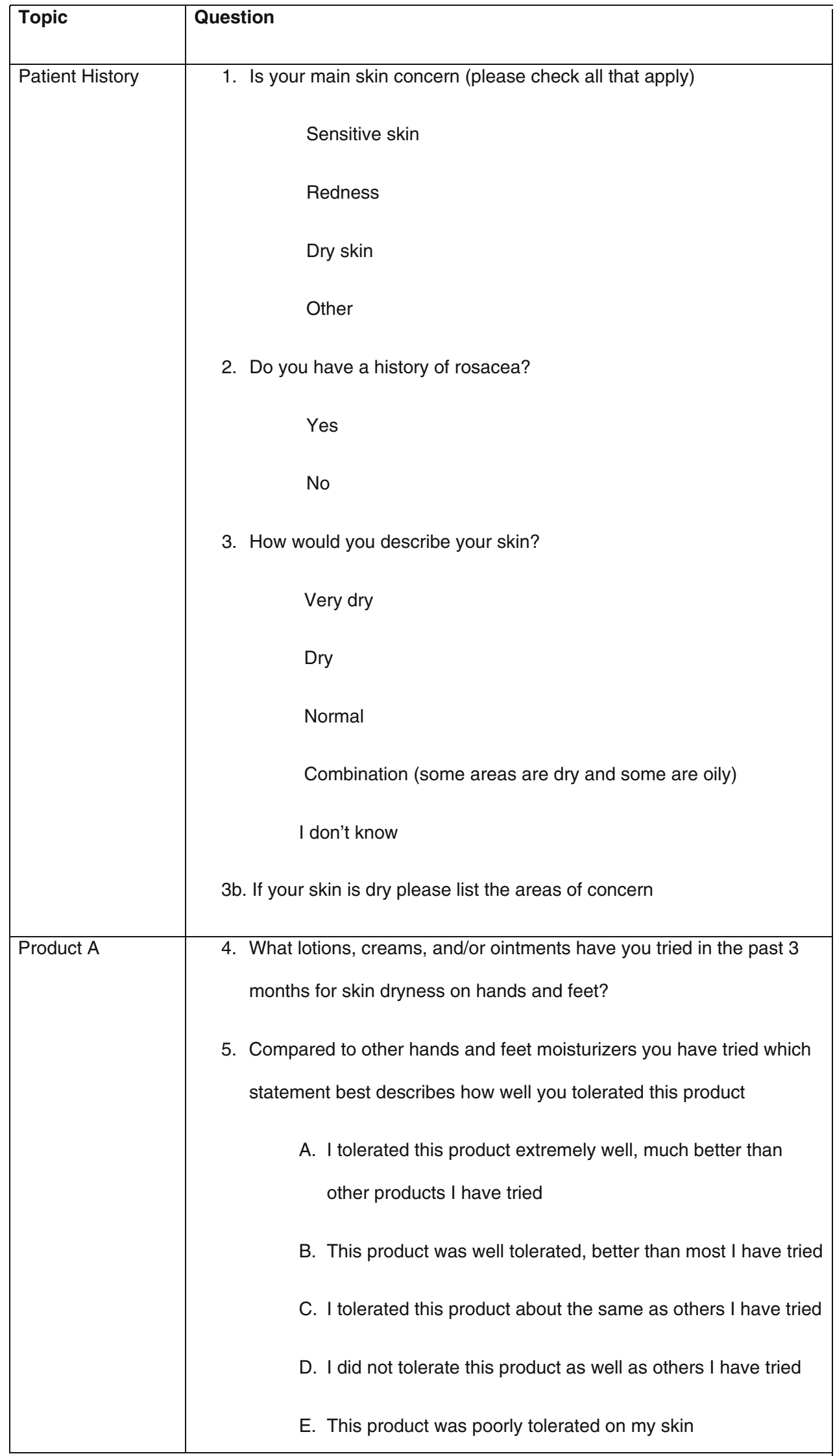

Fig. 1 Skin care management tolerability questionnaire 


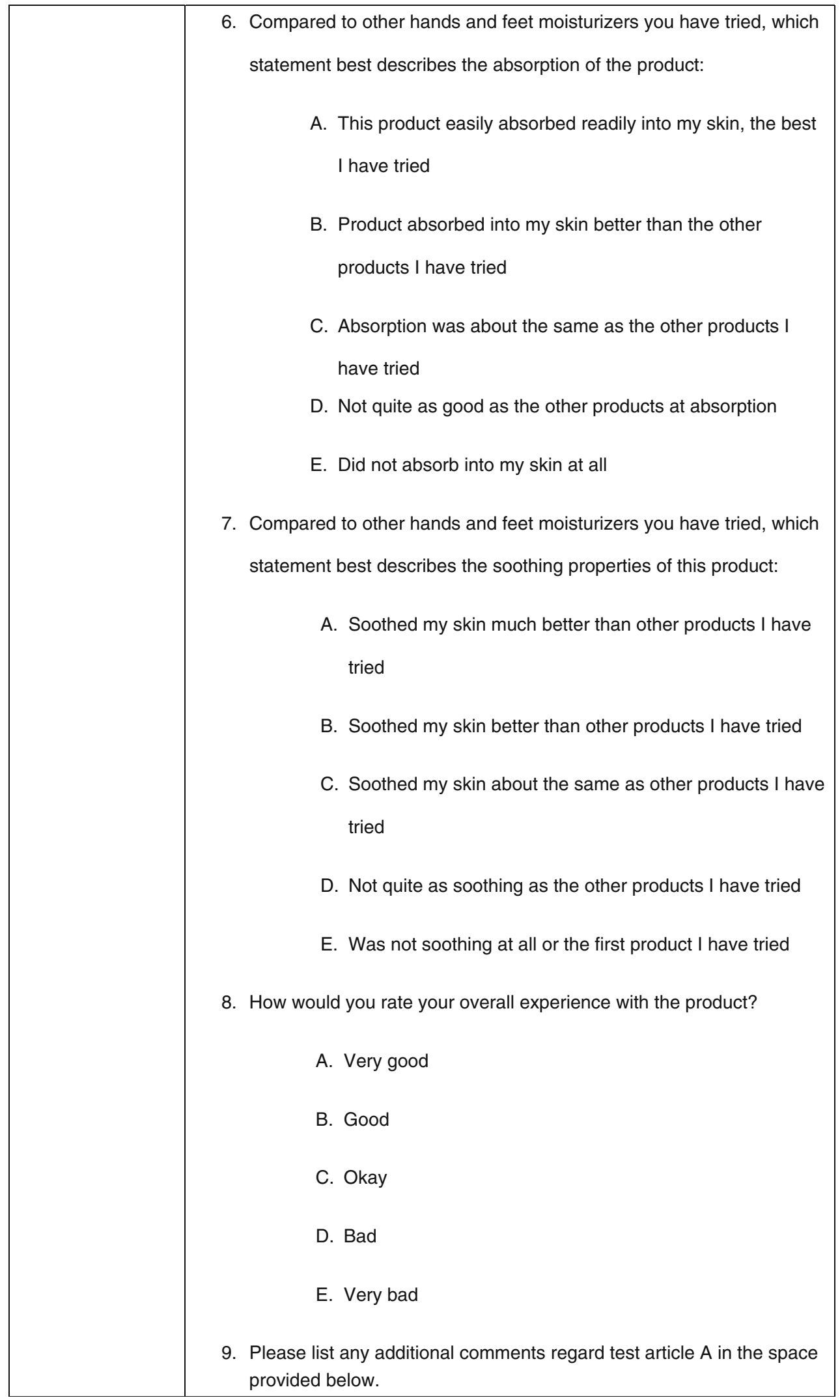

Fig. 1 (continued) 
Fig. 1 (continued)
10. What face lotions and/or creams have you tried in the past 3 months?

11. Compared to other facial moisturizers you have tried, which statement best describes the decrease in redness you noticed when using this product:

A. This product was much better at decreasing the appearance of my redness than others I have tried

B. This product was slightly or somewhat better at decreasing the appearance of my redness than the others I have tried

C. About the Same

D. Not quite as good

E. Did not notice any redness reduction at all

12. Compared to other facial moisturizers you have tried, which statement best describes the amount of dryness relief you experienced with this product:

A. Much better than the others I have tried

B. Somewhat better than the others I have tried

C. Relived my dryness about as well as the other products I have tried

D. Not quite as well

E. No or minimal dryness relief from this product, compared to the others

13. Compared to other facial moisturizers you have tried, which statement best describes the improvement in the overall appearance of my skin while using this product:
A. My skin appeared greatly improved
B. My skin appeared to look somewhat improved
C. My skin appeared to look about the same
D. My skin did not appear to look as good
E. My skin looked worse than while using other products 


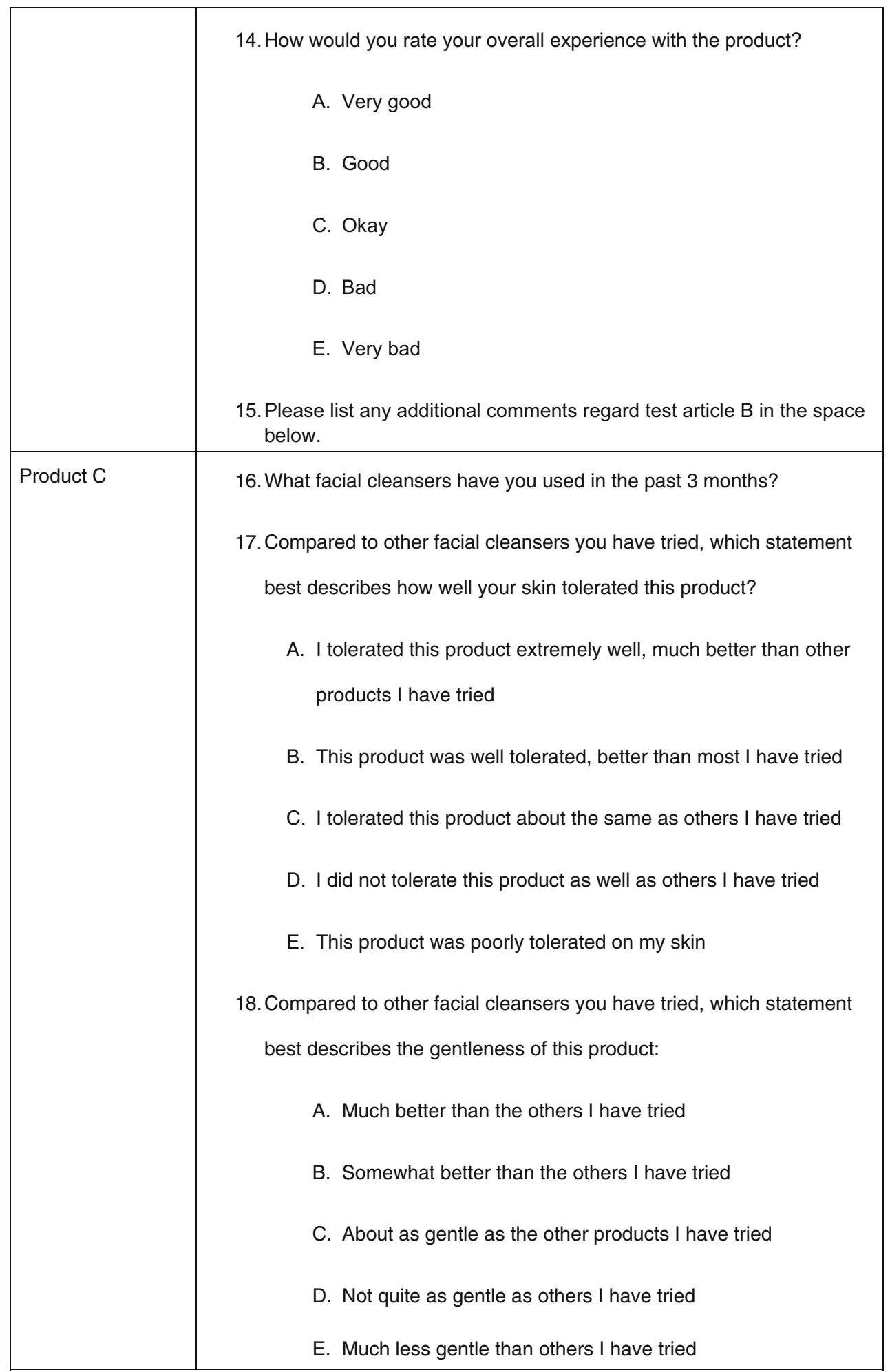

Fig. 1 (continued) 


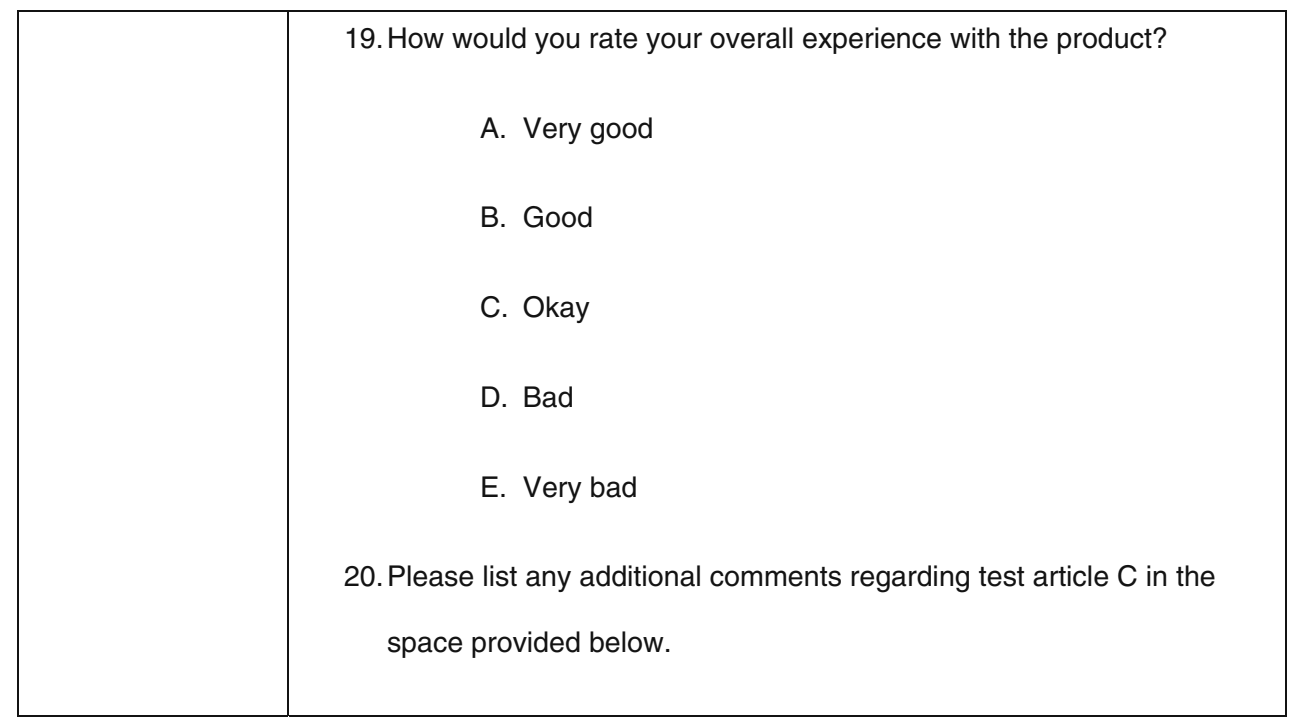

Fig. 1 (continued)

Grading of dermatologic adverse events

Severity of cancer treatment-related skin toxicity was assessed by the research staff using the NCI-CTCAEv3.0. The CTCAE is used to report and grade severity of toxicity in trials of anticancer agents [15]. According to NCICTCAE, grades 2, 3, or 4 require medical intervention [13]. Therefore, subjects with grades 2,3 , or 4 skin toxicities were excluded from this study. Grade 0 refers to no skin toxicity for purposes of this study [13]. Therefore, selection criteria for this study included oncology patients receiving chemotherapy or radiotherapy with a skin toxicity of $0-1$ based on the NCI-CTCAEv3.0 scale.

Assessment of quality of life using the Skindex-16

Skindex is a 16-question quality of life instrument that has been validated to accurately measure the extent patients are bothered by certain skin conditions [14]. Each question asks subjects the degree to which they have been concerned by their specific skin condition in the week prior to administration of the questionnaire. Subjects answer every question with a number ranging from 1 (never bothered) to 6 (always bothered). After Skindex-16 is complete, responses to each item are transformed to a linear scale of 100 , varying from 0 (never bothered) to 100 (always bothered). Thus, each item has a minimum score of 0 and a maximum score of 100. In this study, Skindex-16 is recorded for the baseline visit and the 4-week follow-up interview (16 individual scores) and three groupings of the 16 questionnaire scores as domains: symptoms, emotions, and function. The symptoms (items 1-4), emotions (items 5-11), and function (items 12-16) domains are scores calculated as means ranging from 0 to 100 [15].

\section{Statistical analysis}

Fisher's exact test was used to assess the change in toxicity rates between baseline and follow-up. The Skindex-16 was compared between the baseline visit and follow-up interview using a repeated measures analysis of variance taking into account that some patients had data at two time points and others only had baseline data. This is an overall evaluation of all three test articles used for skin care management and is not specific to each test article. In order to be considered as improved QoL, the mean Skindex-16 score must decrease between the two time points. Analysis is done for the overall scale as well as for the three domains (symptoms, emotions, and function). Fisher's exact test was used to compare product satisfaction across products in patients with rosacea. Fisher's exact test was also used to relate product satisfaction to skin type.

\section{Results}

Of 99 enrolled subjects, 77 completed the four-week assessment. Twenty-two subjects withdrew consent or were lost to follow-up. Two subjects provided incomplete questionnaires resulting in a $n=75$ for analytical purposes. Sample sizes for follow-up results varied due to missing data for certain questionnaire items. 
Table 2 Skindex-16: quality of life subscale domains

\begin{tabular}{|c|c|c|c|c|c|}
\hline \multirow[t]{2}{*}{ Domain } & \multicolumn{2}{|l|}{ Baseline } & \multicolumn{2}{|c|}{ 4-week follow-up visit } & \multirow[t]{2}{*}{$p$ value } \\
\hline & $n$ value & Mean & $n$ value & Mean & \\
\hline Symptoms & 98 & 25.21 & 77 & 17.05 & 0.0085 \\
\hline Emotions & 98 & 32.24 & 77 & 20.22 & 0.0002 \\
\hline Function & 97 & 15.02 & 77 & 9.87 & 0.008 \\
\hline Combined (All) & 98 & 25.05 & 77 & 16.19 & 0.0003 \\
\hline
\end{tabular}

\section{Dermatologic toxicities}

Most subjects $(69 \%, n=75)$ experienced dry skin at baseline and $21 \%(n=75)$ at study completion (4 weeks; $p<0.0001$, Table 1). Of the 11 subjects who presented with HFSR at baseline visit (15\% of 75), three continued to have HFSR present at 4 -week follow-up (4\% of $75, p=0.021)$. Skin rash (dermatitis) decreased from $8 \%(n=75)$ at baseline to $1 \%(n=75)$ at 4 -week follow-up $(p=0.025)$. Few subjects had nail changes (5\% of 74$)$, rash (desquamation; $3 \%$ of 75 ), and acne/acneiform eruptions (4\% of 75 ) at the initial visit, and presence of these toxicities did not significantly change from baseline to 4 week follow-up ( $5 \%$ of $75 p=0.99,0 \%$ of $75 p=0.15,7 \%$ of $75 p=0.32$, respectively).

Dermatology-related quality of life improvement at follow-up

The study population at 4-week follow-up had a mean overall Skindex-16 score of 16.19 , which is lower than baseline of $25.05(p=0.0003)$. Each domain (symptoms, emotions, function) also had a significant decrease in the mean Skindex-16 domain score from baseline to follow-up; symptoms domain value decreased from 25.21 to 17.05 ( $p=0.0085$ ), emotions decreased from 32.24 to 20.22 ( $p=0.0002$ ), function from 15.02 to 9.87 ( $p=0.008$; Table 2 , Fig. 2).

\section{Cancer skin care management survey}

\section{Test article A: skin moisturizer}

Although a high percentage of the 75 evaluable participants $(n=72,96 \%)$ had used other products for chemotherapyassociated dry skin, $63 \%(n=47)$ tolerated test article A better than previous other products. Nearly $30 \%(n=22)$ of participants reported tolerating test article $\mathrm{A}$ about the same as other products previously tried. A majority of subjects $(n=49,65 \%)$ said the test article vanished into the skin better than other previous moisturizers. Most participants $(n=44,59 \%)$ found the test article to soothe the skin better than other products tried for dry skin, and $31 \%(n=23)$ reported the test article soothed the skin about the same as other products tried (Table 3). The majority of subjects,
$79 \%(n=59)$, rated their overall experience with the test article as good or very good (Table 4).

\section{Test article B: face moisturizer}

Of the 77 participants who completed the study, 73 (95\%) subjects used up to three other products to decrease redness and soothe compromised skin on the face within 3 months prior to study initiation. A majority of participants $(n=54$, $70 \%$ ) saw improvement in overall appearance of their facial skin while using test article B compared to other facial moisturizers they had previously tried. Compared to other products used in the past, $70 \%(n=54)$ of subjects reported that their facial skin appeared to look as good or better with use of test article B. Of participants experiencing erythema at study initiation, 55\% $(n=32 / 58)$ of participants experienced a more pronounced decrease in facial erythema with the use of test article B compared to previous products. Moreover, most participants $(n=58,75 \%)$ also experienced enhanced relief of dryness with test article B compared to other products previously used for dry skin of the face (Table 3). A high majority, $88 \%(n=68)$ of participants, rated their overall experience with test article B as good or very good (Table 4).

\section{Test article C: face wash}

Nearly all participants $(n=73,97 \%)$ used one or two other facial cleansers for toxicity related to facial skin within 3 months prior to study initiation. A majority of participants

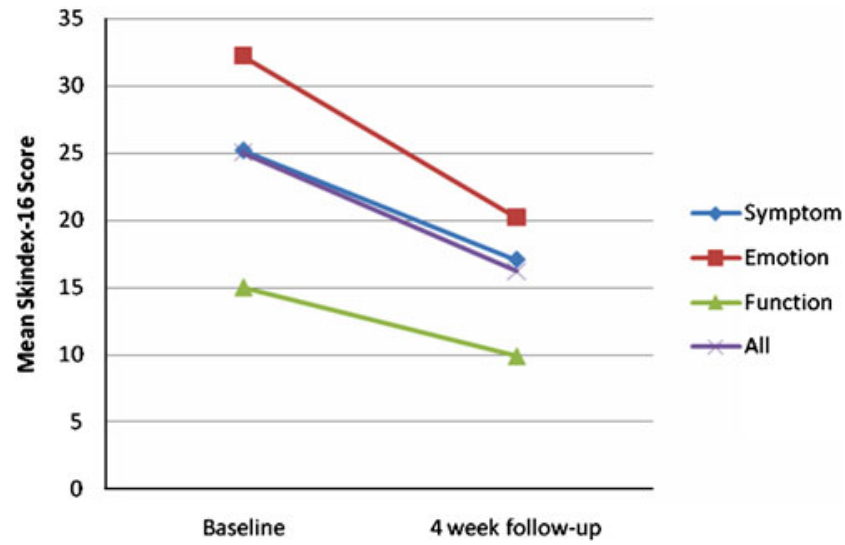

Fig. 2 Change in Skindex-16 scores from baseline to 4-week follow-up 
Table 3 Cancer skin care management survey

\begin{tabular}{|c|c|c|c|c|c|c|c|c|}
\hline & \multicolumn{3}{|c|}{ Test article $\mathrm{A}^{\mathrm{a}}$} & \multicolumn{3}{|l|}{ Test article $\mathrm{B}^{\mathrm{b}}$} & \multicolumn{2}{|l|}{ Test article $\mathrm{C}^{\mathrm{c}}$} \\
\hline & $\begin{array}{l}\text { Tolerability } \\
(n=75)\end{array}$ & $\begin{array}{l}\text { Absorption } \\
(n=75)\end{array}$ & $\begin{array}{l}\text { Soothing } \\
\text { properties } \\
(n=75)\end{array}$ & $\begin{array}{l}\text { Redness } \\
\text { reduction } \\
(n=58)^{\mathrm{d}}\end{array}$ & $\begin{array}{l}\text { Dryness } \\
\text { relief } \\
(n=77)\end{array}$ & $\begin{array}{l}\text { Improvement in } \\
\text { appearance } \\
(n=77)\end{array}$ & $\begin{array}{l}\text { Tolerability } \\
(n=74)\end{array}$ & $\begin{array}{l}\text { Gentleness } \\
(n=75)\end{array}$ \\
\hline $\begin{array}{l}\text { Very good, } \\
\text { good }\end{array}$ & $47(62.67 \%)$ & $49(65.33 \%)$ & $44(58.67 \%)$ & $32(55.17 \%)$ & $58(75.32 \%)$ & $54(70.13 \%)$ & $46(62.16 \%)$ & $50(66.67 \%)$ \\
\hline $\begin{array}{l}\text { About the } \\
\text { same }\end{array}$ & $22(29.33 \%)$ & $14(18.67 \%)$ & $23(30.67 \%)$ & $14(24.24 \%)$ & $11(14.29 \%)$ & $20(25.97 \%)$ & $24(32.43 \%)$ & $24(32.00 \%)$ \\
\hline $\begin{array}{l}\text { Bad, very } \\
\text { bad }\end{array}$ & $6(8.00 \%)$ & $12(16.00 \%)$ & $8(10.67 \%)$ & $12(20.69 \%)$ & $8(10.39 \%)$ & $3(3.90 \%)$ & $4(5.41 \%)$ & $1(1.33 \%)$ \\
\hline
\end{tabular}

${ }^{\text {a }}$ Skin moisturizer

${ }^{\mathrm{b}}$ Facial moisturizer

${ }^{\mathrm{c}}$ Facial wash

${ }^{\mathrm{d}} 41$ patients did not have any redness initially

( $n=46,62 \%$ ) tolerated test article $\mathrm{C}$ better than other facial cleansers. Most participants $(n=50,67 \%)$ rated test article $\mathrm{C}$ as more gentle, and $32 \%(n=24)$ rated product $\mathrm{C}$ as about as gentle compared to other products (Table 3 ). In general, $74 \%(n=56)$ of subjects rated their overall experience with test article $\mathrm{C}$ as good or very good (Table 4 ).

\section{Tolerability for participants with pre-existing rosacea}

Twelve $(16 \%)$ of the evaluable participants $(n=74)$ using test article A reported pre-existing rosacea. A majority of participants ( $n=10,83 \%, 95 \%$ CI $61-95 \%)$ presenting with pre-existing rosacea indicated that test article A was very good or good. Of the 12 subjects with rosacea, 11 indicated that test article B was very good or good $(92 \%, 95 \%$ CI $73-$ $98 \%)$. Of the 12 subjects with rosacea, nine or $75 \%(95 \% \mathrm{CI}$ $51-91 \%$ ) indicated that test article $\mathrm{C}$ was very good or good. A Fisher's exact test to compare cancer skin care management satisfaction by patients with pre-existing rosacea across all three test articles (A, B, and C) indicated similar acceptability ( $p=0.85$, when comparing $83 \%, 92 \%, 75 \%$ ).

\section{Skin type versus product satisfaction}

A correlation was not found by examining skin type (very dry/dry, normal, combination/do not know) versus product satisfaction. Satisfaction with the skin moisturizer (test article A) across skin types $(n=73)$ did not vary by skin type ( $p=0.82$ by Fisher's exact test). Satisfaction with the facial moisturizer (test article B) across skin types was not significant $(n=75, p=0.71)$. Lastly, satisfaction with test article C compared across subject skin types was not different $(p=0.40, n=74)$.

\section{Discussion}

Dermatologic toxicities are common side effects associated with anticancer treatments, and these adverse events often negatively impact a patient's quality of life and willingness to continue with treatment [3]. Management of these events in cancer patients is suboptimal in part due to the lack of products specifically designed for this patient population. In seeking to investigate the tolerability and effects on quality of life for products designed for cancer skin care management, these findings suggest that quality of life, as measured by Skindex-16, improves with products specifically designed for cancer skin care management. Moreover, the severity of skin toxicity graded according to CTCAE decreases within 1-month use of products specifically designed for cancer skin care management, and dermatology quality of life significantly improves as early as 1 month for individual domains (symptoms, emotions, functions).

Table 4 Cancer skin care management survey: overall experience in patients

\begin{tabular}{|c|c|c|c|c|c|c|}
\hline \multirow[t]{2}{*}{ Overall experience } & \multicolumn{2}{|c|}{ Skin moisturizer (test article A) } & \multicolumn{2}{|c|}{ Face moisturizer (test article B) } & \multicolumn{2}{|c|}{ Face wash (test article C) } \\
\hline & $n=75$ & $\%$ & $n=77$ & $\%$ & $n=76$ & $\%$ \\
\hline Very good, good & 59 & 78.67 & 68 & 88.31 & 56 & 73.68 \\
\hline Okay & 12 & 16.00 & 7 & 9.09 & 18 & 23.68 \\
\hline Bad, very bad & 4 & 5.33 & 2 & 2.60 & 2 & 2.63 \\
\hline
\end{tabular}


This study demonstrates that products specifically designed for cancer skin care management improve skinrelated quality of life. Moreover, patients with rosacea and rosacea-like erythematous facial skin are often very sensitive to skin care products that can worsen inflammation and produce irritation [16]. This study demonstrated cancer patients with pre-existing rosacea tolerate test articles A, B, and C. Maintenance of cutaneous integrity during cancer treatments with the use of moisturizers may aid in mitigating dry skin, which may be associated with pruritus and infections, minimizing these comorbidities [17, 18].

In summary, specifically tailored skin care management for cancer patients is well tolerated by patients currently undergoing anticancer therapy or radiation, and a majority of subjects report that such therapies are better than previous regimens used. A majority of participants also report improved dermatology QoL. Previous studies indicate that dermatology QoL rebounds within 4 months of completion of anticancer regiments, making it important for patients to receive interventions during anticancer therapy and the immediate 4-month follow-up period [19].

Advance knowledge of side effects and proactive management approaches have been shown to decrease anxiety, improve adherence to cancer treatment, and improve QoL and patient outcomes [5]. Studies suggest that anticancer therapy side effects are less anxiety provoking when anticipated [20]. If patients engage in anticipatory coping, a mechanism involving "affective and behavioral rehearsal," patients feel in control when toxicities resulting from cancer treatments arise [21]. A cancer skin care management program fulfills patient engagement of anticipatory coping in order to optimally anticipate and control cutaneous side effects associated with anticancer therapy.

Acknowledgments We are grateful to Lindi ${ }^{\circledR}$ Skin for providing test articles. M.E.L. is supported by a Zell Scholarship from the Robert H Lurie Comprehensive Cancer Center and a Dermatology Foundation Career Development Award.

Open Access This article is distributed under the terms of the Creative Commons Attribution Noncommercial License which permits any noncommercial use, distribution, and reproduction in any medium, provided the original author(s) and source are credited.

\section{References}

1. Lacouture M, West D, Tigue C, Knox K, Bennett C (2008) Cutaneous toxicities of targeted cancer therapies. Community Oncology 5(7):413-414

2. Hackbarth M, Haas N, Fotopoulou C, Lichtenegger W, Sehouli J (2008) Chemotherapy-induced dermatological toxicity: frequencies and impact on quality of life in women's cancers. Results of a prospective study. Support Care Cancer 16(3):267-273
3. O'Keeffe P, Parrilli M, Lacouture M (2006) Toxicity of targeted therapy: focus on rash and other dermatologic side effects. Oncol Nurses Ed 20:1-6

4. Wagner L, Lacouture M (2007) Dermatologic toxicities associated with EGFR inhibitors: the clinical psychologist's perspective. Impact on health-related quality of life and implications for clinical management of psychological sequelae. Oncology (Williston Park) 21(11 Suppl 5):34-36

5. Gandhi M, Oishi K, Zubal B. Unanticipated toxicities from anticancer therapies: survivors' perspectives.Support Care in Cancer (in press)

6. Autier J, Escudier B, Wechsler J, Spatz A, Robert C (2008) Prospective study of the cutaneous adverse effects of sorafenib, a novel multikinase inhibitor. Arch Dermatol 144(7): $886-892$

7. Richardson L, Wang W, Hartzema A, Wagner S (2007) The role of health-related quality of life in early discontinuation of chemotherapy for breast cancer. Breast J 13(6):581-587

8. Susser W, Whitaker-Worth D, Grant-Kels J (1999) Mucocutaneous reactions to chemotherapy. J Am Acad Dermatol 40(3):367398, quiz 99-400

9. Lynch T, Kim E, Eaby B, Garey J, West D, Lacouture M (2007) Epidermal growth factor receptor inhibitor-associated cutaneous toxicities: an evolving paradigm in clinical management. Oncologist 12(5):610-621

10. McQuestion M (2006) Evidence-based skin care management in radiation therapy. Semin Oncol Nurs 22(3):163-173

11. Bolderston A, Lloyd N, Wong R, Holden L, Robb-Blenderman L (2006) The prevention and management of acute skin reactions related to radiation therapy: a systematic review and practice guideline. Support Care Cancer 14(8):802-817

12. Lindi ${ }^{\circledR}$ Skin (2008) http://lindiskin.com/

13. CTC v2.0 and common terminology criteria for adverse events v3.0 (CTCAE). (2009) http://ctep.cancer.gov/reporting/index.html

14. Chren M, Lasek R, Sahay A, Sand L (2001) Measurement properties of Skindex-16: a brief quality-of-life measure for patients with skin diseases. J Cutan Med Surg 5(2):105-110

15. Basch E, Iasonos A, McDonough T, Barz A, Culkin A, Kris M et al (2006) Patient versus clinician symptom reporting using the National Cancer Institute Common Terminology Criteria for Adverse Events: results of a questionnaire-based study. Lancet Oncol 7(11):903-909

16. Weber T, Ceilley R, Buerger A, Kolbe L, Trookman N, Rizer R et al (2006) Skin tolerance, efficacy, and quality of life of patients with red facial skin using a skin care regimen containing Licochalcone A. J Cosmet Dermatol 5(3):227-232

17. Robert C, Soria JC, Spatz A, Le Cesne A, Malka D, Pautier P et al (2005) Cutaneous side-effects of kinase inhibitors and blocking antibodies. Lancet Oncol 6(7):491-500

18. Eilers RE Jr, Gandhi M, Patel JD, Mulcahy MF, Agulnik M, Hensing T et al (2010) Dermatologic infections in cancer patients treated with epidermal growth factor receptor inhibitor therapy. J Natl Cancer Inst 102(1):47-53

19. Fairclough D, Fetting J, Cella D, Wonson W, Moinpour C (1999) Quality of life and quality adjusted survival for breast cancer patients receiving adjuvant therapy. Eastern Cooperative Oncology Group (ECOG). Qual Life Res 8(8):723-731

20. Frith H, Harcourt D, Fussell A (2007) Anticipating an altered appearance: women undergoing chemotherapy treatment for breast cancer. Eur J Oncol Nurs 11(5):385-391

21. Burish T, Snyder S, Jenkins R (1991) Preparing patients for cancer chemotherapy: effect of coping preparation and relaxation interventions. J Consult Clin Psychol 59(4):518-525 